\title{
Test of rotational invariance in $\beta$ decay
}

\author{
H. W. Wilschut ${ }^{1}$ A. Sytema ${ }^{1}$
}

Published online: 29 June 2016

(C) The Author(s) 2016. This article is published with open access at Springerlink.com

\begin{abstract}
The possibility of violation of rotational invariance in $\beta$ decay is considered. Such violation would imply Lorentz invariance violation. Recent developments in experiment and theory are discussed.
\end{abstract}

Keywords Lorentz violation $\cdot \beta$-decay

PACS 11.30.Cp $\cdot 24.80 .+\mathrm{y} \cdot 23.40 . \mathrm{Bw}$

\section{Introduction}

In 1956, Lee and Yang noted in their seminal paper that conservation of parity and angular momentum had not been tested in the weak interaction. While shortly after parity conservation was shown to be violated, rotational invariance remains poorly tested in the weak interaction. With C, P, T and CP violated, the weak interaction is an important portal to search for physics beyond the Standard Model. CPT violation is a relevant possibility in the unification of the Standard Model and General Relativity. CPT violation implies Lorentz Violation (LV) [1]. In $\beta$ decay it could mean, for example, a preferred direction of $\beta$ emission. We have performed a direct test of rotational invariance in $\beta$ decay by observing whether the $\beta$-decay rate of polarized nuclei depends on the polarization direction. Violation of rotational invariance is a manifestation of LV. Even in the absence of a complete theory different $\mathrm{LV}$ violating observables can be interpreted in common terms using an

This article is part of the Topical Collection on Proceedings of the 6th International Symposium on Symmetries in Subatomic Physics (SSP 2015), Victoria, Canada, 8-12 June 2015

H. W. Wilschut wilschut@kvi.nl

1 Van Swinderen Institute for Particle Physics and Gravity, Nijenborgh 4, 9747 AG Groningen, The Netherlands 
effective field theory such as the Standard Model Extension [2]. In a recent experiment we improved the limit on rotational invariance by an order of magnitude to $10^{-4}$. We will discuss theoretical framework focused on allowed $\beta$ decay and apply it to the result of our experiment.

\section{Theoretical framework}

A rather general theoretical framework for LV in weak interactions was given in Refs. [3, 4] for $\beta$ decay and in Ref. [5] for orbital electron capture. They describe the effects of a modified low-energy $W$-boson propagator

$$
\left\langle W^{\mu+} W^{\nu-}\right\rangle=-i\left(g^{\mu \nu}+\chi^{\mu \nu}\right) / M_{W}^{2},
$$

where $g^{\mu \nu}$ is the Minkowski metric and $\chi^{\mu \nu}$ is a general Lorentz-violating (complex, possibly momentum-dependent) tensor, which can be expressed in terms of parameters defined in the Standard Model Extension of Kostelecký and co-workers [2]. The present limits of $\chi^{\mu \nu}$ were recently discussed in [6]. Measurements in allowed $\beta$ decay can be seen as an extension of $\beta-v$ correlations in the formalism of Jackson, Treiman and Wyld (JTW) [7]. Considering only $\beta$-particle emission and polarization of the parent nucleus, and using Standard Model values for the JTW parameters, one obtains for allowed pure Fermi $(F)$ and Gamow-Teller $(G T)$ transitions the following decay rates [8]

$$
\begin{aligned}
d W_{F}= & d W_{0}\left[1+2 \chi_{r}^{00}+2 \chi_{r}^{0 l} \beta^{l}\right] \\
d W_{G T}= & d W_{0}\left[1-\frac{2}{3} \chi_{r}^{00}+\frac{2}{3}\left(\tilde{\chi}_{i}^{l}+\chi_{r}^{0 l}\right) \beta^{l}\right. \\
& +A \frac{J^{k}}{J}\left(\tilde{\chi}_{i}^{k}+\beta^{l}\left\{\left(1-\chi_{r}^{00}\right) \delta_{k l}+\chi_{r}^{l k}\right\}\right) \\
& \left.+A \chi_{i}^{l 0}\left(\frac{\langle\vec{J}\rangle}{J} \times \vec{\beta}\right)^{l}\right] .
\end{aligned}
$$

Here $l, k$ are the spatial directions $(1,2,3)$. The velocity of the $\beta$ particle is given as $\vec{\beta}$ with components $\beta^{l}$, similarly the spin $\vec{J}$ of the parent nucleus. $A$ is the $\beta$ asymmetry "Wu") parameter. The parameters $\chi^{\mu \nu}$ can have a real and imaginary component indicated by subscripts $r, i$. At the low energies of $\beta$ decay one may use [3] the approximation $\left(\chi^{\mu \nu}\right)^{*}=\chi^{\nu \mu}$ so that $\tilde{\chi}_{i}^{1}=2 \chi^{23}, \tilde{\chi}_{i}^{2}=2 \chi_{i}^{13}$, and $\tilde{\chi}_{i}^{3}=2 \chi^{12}$. The parameters $\chi^{\mu \nu}$ are defined in the particle frame. They need to be transformed to a fixed frame for which it is custom to take the Sun centered frame. For a fixed laboratory setup the changes in velocity with respect to this frame are so small that they can be ignored. (This is not the case for $\beta$ decay of high-energy $(\gamma \gg 1)$ radioactive-particles.) Current limits on $\chi$ components are at least below $10^{-3}$ and in the absence of fine tuning at the level of $10^{-6}$ [9]. The only components not measured until now are $\chi_{i}^{l 0}$. This is mainly because this component requires a measurement of the vector product $(\vec{J} \times \vec{\beta})$ as a function of its orientation in absolute space [10]. 

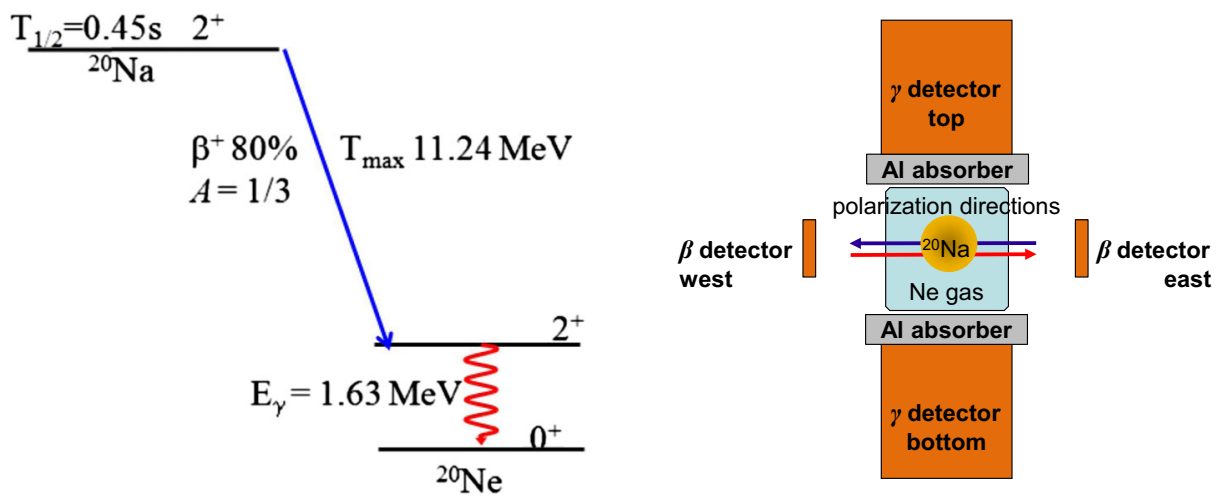

Fig. 1 Left: relevant details of the level scheme of ${ }^{20} \mathrm{Na} \beta$ decay. Right: schematic setup of the experiment

\section{Principle of measurement}

To obtain precise limits on the various $\chi$ components, accurate measurements that are robust against systematical errors are necessary. From (1) and (2) one can derive count rate asymmetries by measuring $\beta$-particle emission in specific but opposite directions. These asymmetries depend on $\chi$. In the present experiment, we studied a $G T$ decay where only the spin is reversed. The decay rate itself is obtained by measuring a $\gamma$ decay of the daughter nucleus, thereby integrating over all directions of the $\beta$ particle. Therefore, this measurement is sensitive to $\tilde{\chi}_{i}^{k}$. We use ${ }^{20} \mathrm{Na}$ for this experiment. Its relevant properties are shown in Fig. 1 together with a schematic setup of the experiment. The sample was polarized in the east-west direction ( $k=1$ in 2 ). It is further assumed that $P=\left\langle J^{1}\right\rangle / J$, while $\left\langle J^{2,3}\right\rangle=0$. We measure two asymmetries in parallel. The first one determines LV by

$$
\frac{W^{\uparrow}-W^{\downarrow}}{W^{\uparrow}+W^{\downarrow}}=A P \tilde{\chi}_{i}^{1}=\frac{\tau^{\downarrow}-\tau^{\uparrow}}{\tau^{\downarrow}+\tau^{\uparrow}},
$$

where the arrows indicate the opposite polarization directions. The third part of this equation is to indicate that instead of measuring rates, we measure lifetimes $(W \propto 1 / \tau)$. The second asymmetry that we measure derives the polarization from the parity violating term $(\vec{J} \cdot \vec{\beta})$ by measuring the $\beta$-decay rate, $R$, in approximately identical $\beta$ detectors, one east $(E)$ and one west $(W)$. We use

$$
\frac{\sqrt{R_{E}^{\uparrow} R_{W}^{\downarrow}}-\sqrt{R_{E}^{\downarrow} R_{W}^{\uparrow}}}{\sqrt{R_{E}^{\uparrow} R_{W}^{\downarrow}}+\sqrt{R_{E}^{\downarrow} R_{W}^{\uparrow}}} \approx A P .
$$

In determining the polarization the LV terms have been neglected, they only contribute in higher order of $\chi$.

\section{Experimental results}

Figure 2 shows the result of measurements of the $\beta$ asymmetry (4). It has been obtained by combining consecutive periods of $4 \mathrm{~s}$ with opposite polarization. In the first 2 seconds a constant beam of ${ }^{20} \mathrm{Na}$ is entering the fiducial volume of a gas cell filled with ${ }^{20} \mathrm{Ne}$. The maximum polarization $P$ that could be reached was about $50 \%$. In the last 2 seconds when 


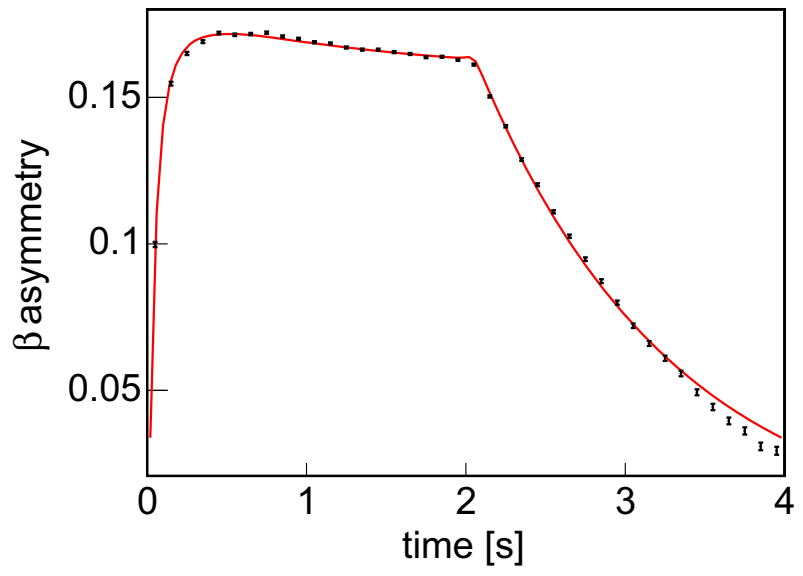

Fig. 2 The experimental $\beta$ asymmetry as defined in (4)

no new ${ }^{20} \mathrm{Na}$ particles enter the setup, the polarization is seen to decrease. The $\beta$ asymmetry can be well described with a three parameter function. The time dependent polarization results from the dynamics of stopping and neutralization of fast particles in a buffer gas and the chemistry due to contaminants [11]. A time dependent description of the polarization is important to interpret the measured lifetimes in the $2 \mathrm{~s}$ periods in terms of the asymmetry defined in (3). If there is an observable value for $\tilde{\chi}_{i}^{1}$, then the asymmetry should show a variation with sidereal time. The laboratory rotates in the sun-centered frame by $180^{\circ}$ in half a sidereal day changing the sign of the correlation. Making the transformation $\chi^{\mu \nu}$ to parameters $X^{\mu \nu}$ in the fixed frame one finds

$$
\tilde{\chi}_{i}^{1}=\tilde{X}_{i}^{1} \cos (\Omega t+\phi)+\tilde{X}_{i}^{2} \sin (\Omega t+\phi),
$$

where $\Omega$ is the sidereal frequency and $\phi$ is a phase to obtain the proper orientation at $t=0$ in the sun-centered frame. We find no significant oscillation in our experiment. This allows us to set limits on $\tilde{X}_{i}^{1}$ and $\tilde{X}_{i}^{2}$, which means limits on $X_{i}^{23}$ and $X_{i}^{13}$. The analysis will be discussed in a forthcoming paper. The preliminary result is $\left|X_{i}^{23}\right|,\left|X_{i}^{13}\right|<3 \times 10^{-4}$ at 90 $\%$ confidence.

\section{Conclusion and outlook}

The possibility of Lorentz violation needs to be considered for each interaction separately. The weak interaction is still poorly tested. We have shown that variations of the $\beta$-decay rate as function of the absolute direction of the $\beta$ particle, or the spin direction of the parent nucleus is a way to measure quantitatively Lorentz violation. Here an experiment on a Gamow-Teller transition was discussed. Our result and the results from other experiments, in particular those of forbidden transitions [4], restrict the values of $\chi^{\mu \nu}$ in various ways, depending on the extent of fine tuning. (For an overview see [6].) The only unknown value without any limit is $\chi_{i}^{l 0}$. A program to search for LV concurrently to other searches for new physics in $\beta$ decay was suggested recently in [8]. In particular the weak decay of fast moving particles can lead to strict limits. 
Acknowledgments This research was financially supported by the Stichting voor Fundamenteel Onderzoek der Materie (FOM) under Programme 114 (TRI $\mu$ P) and "FOM projectruimte" 08PR2636-1.

Open Access This article is distributed under the terms of the Creative Commons Attribution 4.0 International License (http://creativecommons.org/licenses/by/4.0/), which permits unrestricted use, distribution, and reproduction in any medium, provided you give appropriate credit to the original author(s) and the source, provide a link to the Creative Commons license, and indicate if changes were made.

\section{References}

1. Greenberg, O.W.: Phys. Rev. Lett. 89, 231602 (2002). doi:10.1103/PhysRevLett.89.231602

2. Colladay, D., Kostelecký, V.A.: Phys. Rev. D 58, 116002 (1998). doi:10.1103/PhysRevD.58.116002

3. Noordmans, J.P., Wilschut, H.W., Timmermans, R.G.E.: Phys. Rev. C 87, 055502 (2013). doi:10.1103/PhysRevC.87.055502

4. Noordmans, J.P., Wilschut, H.W., Timmermans, R.G.E.: Phys. Rev. Lett. 111, 171601 (2013). doi:10.1103/PhysRevLett.111.171601

5. Vos, K.K., Wilschut, H.W., Timmermans, R.G.E.: Phys. Rev. C 91, 038501 (2015). doi:10.1103/PhysRevC.91.038501

6. Vos, K.K., Wilschut, H.W., Timmermans, R.G.E.: Rev. Mod. Phys. 87, 1483 (2015). doi:10.1103/RevModPhys.87.1483

7. Jackson, J., Treiman, S., Jr., H.W.: Nuclear Physics 4, 206 (1957). doi:10.1016/0029-5582(87)90019-8

8. Vos, K.K., Wilschut, H.W., Timmermans, R.G.E.: Phys. Rev. C 92, 052501 (2015). doi:10.1103/PhysRevC.92.052501

9. Vos, K.K., Wilschut, H.W., Timmermans, R.G.E.: Rev. Mod. Phys. 87, 1483 (2015). doi:10.1103/RevModPhys.87.1483

10. Vos, K.K., Wilschut, H.W., Timmermans, R.G.E.: Phys. Rev. C 92, 052501 (2015). doi:10.1103/PhysRevC.92.052501

11. Sytema, A. et al.: Nucl. Instrum. Meth. A 822(77) (2016). doi:10.1016/j.nima.2016.03.086 